\title{
Diabetes as an independent predictor of high atherosclerotic burden assessed by coronary computed tomography angiography: the coronary artery disease equivalent revisited
}

\author{
Pedro de Araújo Gonçalves • Hector M. Garcia-Garcia • Maria Salomé Carvalho • \\ Helder Dores · Pedro Jeronimo Sousa - Hugo Marques • Antonio Ferreira • \\ Nuno Cardim - Rui Campante Teles - Luís Raposo - Henrique Mesquita Gabriel • \\ Manuel Almeida • Ana Aleixo - Miguel Mota Carmo - Francisco Pereira Machado • \\ Miguel Mendes
}

Received: 10 October 2012/Accepted: 5 December 2012/Published online: 13 December 2012

(C) Springer Science+Business Media Dordrecht 2012

\begin{abstract}
To study the prevalence and severity of coronary artery disease (CAD) in diabetic patients. (2) To provide a detailed characterization of the coronary atherosclerotic burden, including the localization, degree of stenosis and plaque composition by coronary computed tomography angiography (CCTA). Single center prospective registry including a total of 581 consecutive stable patients (April 2011-March 2012) undergoing CCTA (Dual-source CT) for the evaluation of suspected CAD without previous myocardial infarction or revascularization procedures. Different coronary plaque burden indexes and plaque type and distribution patterns were compared between patients with $(\mathrm{n}=85)$ and without diabetes $(\mathrm{n}=496)$. The prevalence of CAD (any plaque; $74.1 \mathrm{vs.}$ $56 \% ; p=0.002)$ and obstructive CAD $(\geq 50 \%$ stenosis; 31.8 vs. $10.3 \% ; p<0.001)$ were significantly higher in diabetic patients. The remaining coronary atherosclerotic burden indexes evaluated (plaque in LM-3v-2v with prox. LAD; SIS; SSS; CT-LeSc) were also significantly higher in
\end{abstract}

P. de Araújo Gonçalves $(\bowtie) \cdot$ M. S. Carvalho $\cdot$ H. Dores ·

P. J. Sousa · A. Ferreira - R. C. Teles · L. Raposo

H. M. Gabriel · M. Almeida - A. Aleixo · M. Mendes

Cardiology Department, Centro Hospitalar Lisboa Ocidental,

Av. Prof. Reinaldo dos Santos, 2790-134 Carnaxide, Lisbon,

Portugal

e-mail: paraujogoncalves@yahoo.co.uk

P. de Araújo Gonçalves · H. Marques · A. Ferreira ·

N. Cardim - F. P. Machado

Hospital da Luz, Lisbon, Portugal

P. de Araújo Gonçalves · A. Aleixo · M. M. Carmo

CEDOC - Chronic Diseases Research Center-FCM-NOVA,

Lisbon, Portugal

H. M. Garcia-Garcia

Thoraxcenter, Erasmus MC, Rotterdam, The Netherlands diabetic patients. In the per segment analysis, diabetics had a higher percentage of segments with plaque in every vessel (2.6/13.1/7.5/10.5\% for diabetics vs. 1.4/7.1/3.3/ $4.4 \%$ for nondiabetics for LM, LAD, LCx, RCA respectively; $p<0.001$ for all) and of both calcified (19.3 vs. $9.2 \%, p<0.001)$ and noncalcified or mixed types $(14.4$ vs. $7.0 \% ; p<0.001)$; the ratio of proximal-to-distal relative plaque distribution (calculated as LM/proximal vs. $\mathrm{mid} /$ distal/branches) was lower for diabetics ( $0.75 \mathrm{vs.} 1.04$; $p=0.009)$. Diabetes was an independent predictor of $\mathrm{CAD}$ and was also associated with more advanced CAD, evaluated by indexes of coronary atherosclerotic burden. Diabetics had a significantly higher prevalence of plaques in every anatomical subset and for the different plaque composition. In this report, the relative geographic distribution of the plaques within each subgroup, favored a more mid-to-distal localization in the diabetic patients.

Keywords Diabetes - Coronary artery disease . Atherosclerotic burden - Coronary CT angiography

\section{Introduction}

Patients with diabetes are considered to be at an increased risk of cardiovascular events and therefore it has been recommended by many guidelines a more aggressive management of this subset of patients, especially for those with established cardiovascular disease [1,2].

By contrast, on a primary prevention unselected population level, some of the preventive measures for diabetic patients, like the use of antiplatelets, have failed to demonstrate a clear clinical benefit [3] and are no longer recommended in the absence of clinical evidence of atherosclerotic disease [4]. The reason for the lack of 
benefit of aspirin in diabetic patients is likely related to the fact that diabetic patients represents an heterogeneous subset in what concerns the prevalence and severity of atherosclerotic coronary burden.

This illustrates the need for risk stratification of diabetic patients to identify the ones that can benefit from a more aggressive management at earlier stages. This is an opportunity for noninvasive imaging modalities, such as coronary computed tomography angiography (CCTA), which provides a detailed and comprehensive evaluation of the presence and extent of coronary artery disease (CAD), and can play an important role identifying the diabetic patients that could benefit from a more aggressive prevention of cardiovascular events.

Since CCTA is used mainly as a gatekeeper for the exclusion of significant CAD [5], most of the referred patients are at low to intermediate risk, this provides a good setting to study atherosclerotic disease at an earlier stage.

Therefore the aim of this study is two-folded:

1. To study the prevalence and severity of CAD in diabetic patients at earlier stages of CAD, to further evaluate the concept of CAD equivalent.

2. To provide a detailed characterization of the coronary atherosclerotic burden in diabetic patients, using the comprehensive information derived from CCTA on the localization, degree of stenosis and plaque composition.

\section{Methods}

Population

Single center prospective registry, including a total of 772 consecutive patients undergoing CCTA (with Dual source CT), from April 2011 to March 2012.

Patients were excluded if: (1) previous history of myocardial infarction and/or revascularization procedures ( $\mathrm{n}=70)$; (2) referred for Cardiac CT for other indications than the evaluation of possible CAD (cardiac CT for atrial fibrillation ablation or transcatheter aortic valve implantation procedures; $n=88$ ); (3) referred for suspected acute coronary syndromes $(\mathrm{n}=24)$; $(4)$ with atrial fibrillation or other significant arrhythmias during scan acquisition that compromised image quality $(n=9)$. This resulted in a $24.7 \%$ of the total population being excluded.

For the purpose of this study, 581 stable patients referred for suspected CAD were included in the context of: (1) Previous equivocal or inconclusive stress tests or discordant with the clinical evaluation $(\mathrm{n}=417,71.8 \%)$; (2) Cardiac CT as 1st line investigation of possible CAD $(\mathrm{n}=136,23.4 ; \%) ; 3$ ) Preoperative CAD assessment prior to noncoronary valvular or aortic surgery $(\mathrm{n}=17$; $2.9 \%$ ); (4) Evaluation of possible CAD in cardiomyopathies (Dilated or Hypertrophic) $(\mathrm{n}=11 ; 1.9 \%)$ (Fig. 1: Patient selection and study design).

The study was approved by the local ethics committee and all patients gave a written informed consent.

A detailed medical history by means of a risk factors questionnaire was obtained from the patients to assess for the presence of: (1) Diabetes mellitus (defined as a fasting glucose level of $\geq 7 \mathrm{mmol} / \mathrm{l}$ or the need for insulin or oral hypoglycemic agents) [6]; (2) Dyslipidemia (defined as a total cholesterol level $\geq 5 \mathrm{mmol} / \mathrm{l}$ or treatment with lipidlowering drugs) [7]; (3) Hypertension (defined as blood pressure $\geq 140 / 90 \mathrm{~mm} \mathrm{Hg}$ or the use of antihypertensive medication) [8]; (4) Obesity (body mass index $\geq 30 \mathrm{~kg} / \mathrm{m}^{2}$ ); (5) positive family history of premature CAD [defined as the presence of CAD in first-degree relatives younger than 55 (male) or 65 (female) years of age] [9]; (6) smoking (defined as previous $<1$ year) or current smoker.

Pre-test probability of CAD was determined using both the Diamond and Forrester extended CAD consortium method (DF-CAD consortium model) [10] and the Morise score [11]. The cardiovascular risk was assessed with the HeartScore [4]. For the DF-CAD consortium probability model, as the CAD probability and CV risk of our population was shifted to lower probability (less that $2 \%$ had a $\geq 70 \%$ DF probability), the DF-CAD consortium model categories $\geq 30-70 \%$ and $\geq 70 \%$ were gathered in a intermediate to high $(\geq 30 \%)$ probability group. For the Morise, the original described cut-off points (for low, intermediate and high probability) were used, and for the HeartScore the established high risk cut-off of $\geq 5 \%$ was used.

Scan protocol and image reconstruction

All scans were performed with a dual-source scanner (Somatom Definition, Siemens Medical, Germany), with the patient in dorsal decubitus and in deep inspiration breath-hold. Sublingual nitroglycerin was administered to all patients except when contraindicated and intravenous metoprolol (5 mg, with a titration dose up to $20 \mathrm{mg}$ ) was administered in patients with heart rate $>65$ beats $/ \mathrm{min}$.

During the scan acquisition, a bolus of iodinated contrast (Visipaque, GE Healthcare, USA) was injected at a $6 \mathrm{ml} / \mathrm{s}$ infusion rate, followed by a $50-\mathrm{ml}$ saline flush. The dose of contrast was calculated according to the following formula: (acquisition time $+6 \mathrm{~s}$ delay) $\mathrm{x}$ flow $(6 \mathrm{ml} / \mathrm{s})$. Contrast timing was performed to optimize uniform contrast enhancement of the coronary arteries.

Dose reduction strategies-including electrocardiogram-gated tube current modulation, reduced tube voltage, and prospective axial triggering-were used whenever 
Fig. 1 Patient selection and study design. $C A D$ coronary artery disease, $T A V I$ transcatheter aortic valve implantation, $a F i b$ atrial fibrillation, $M I$ myocardial infarction, $C A B G$ coronary artery bypass grafting, $P C I$ percutaneous coronary intervention, $A C S$ acute coronary syndromes

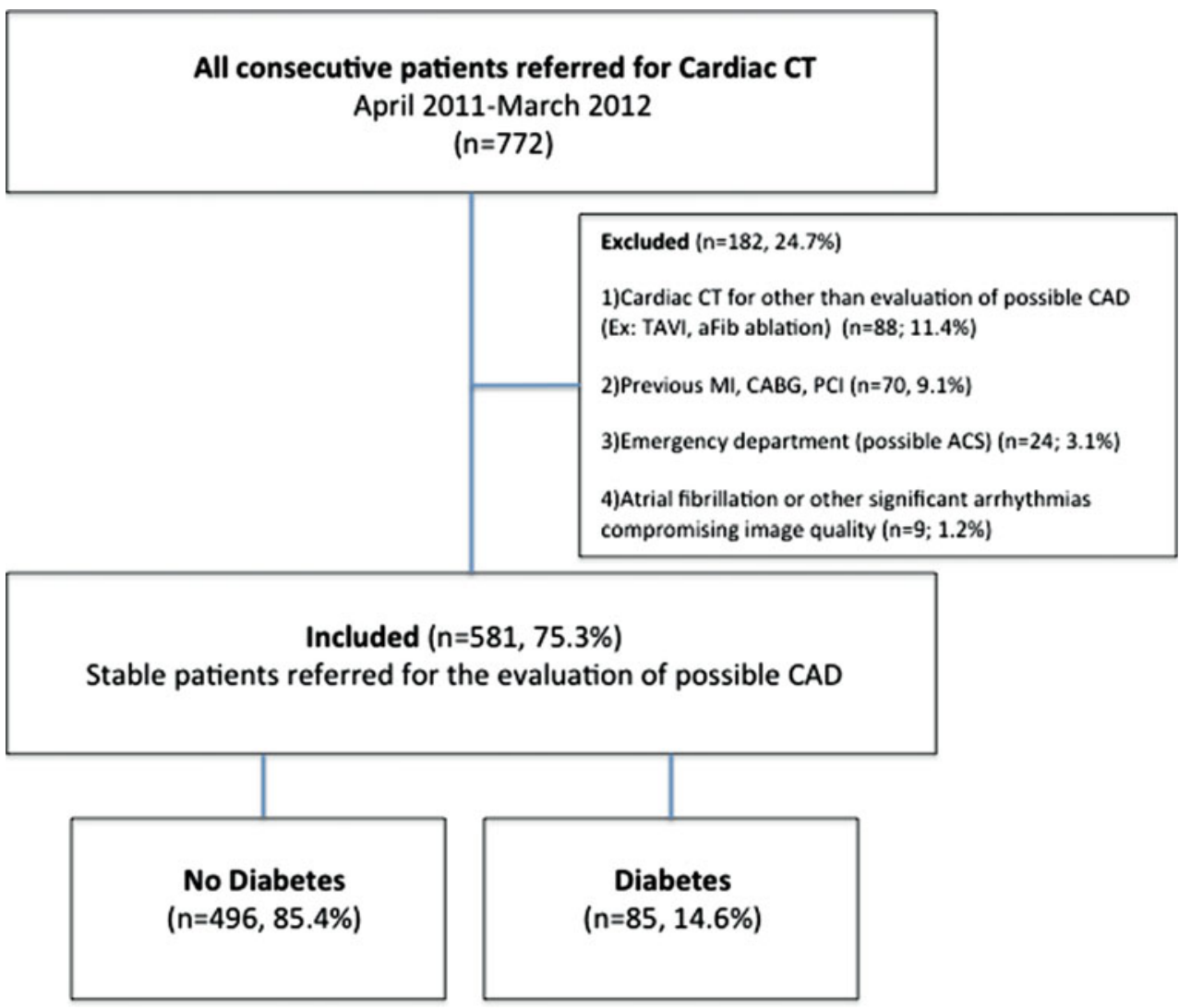

feasible. Mean estimated radiation dose was $4.6 \pm$ $3.7 \mathrm{mSv}$, contrast dose was $98.9 \pm 14.4 \mathrm{ml}$ and heart rate was $65.6 \pm 10.6 \mathrm{bpm}$.

Transaxial images were reconstructed with a temporal resolution of $83 \mathrm{~ms}$ and slice thickness of $0.75 \mathrm{~mm}$ with $0.4 \mathrm{~mm}$ increments.

Post-processing was carried out using Circulation ${ }^{\circledR}$ software, with multiplanar reconstructions, maximum intensity projection and volume rendering technique.

Coronary artery analysis

All scans were analyzed in the same session by both a cardiologist and a radiologist with Level III-equivalent experience. The Society of Cardiovascular Computed Tomography (SCCT) recommended classification was used regarding segmentation (16 segments), stenosis severity $(<25 ; 25-49$; 50-69; 70-99; $100 \%)$ and plaque composition (calcified, noncalcified, mixed plaque) [12]. In each coronary artery segment, coronary atherosclerosis was defined as tissue structures $>1 \mathrm{~mm}^{2}$ that existed either within the coronary artery lumen or adjacent to the coronary artery lumen that could be discriminated from surrounding pericardial tissue, epicardial fat, or the vessel lumen itself. [13] Coronary atherosclerotic lesions were quantified for stenosis by visual estimation. Percent obstruction of coronary artery lumen was based on a comparison of the luminal diameter of the segment exhibiting obstruction to the luminal diameter of the most normal-appearing site immediately proximal to the plaque.

In the detailed per segment analysis, for the distribution of plaque on the 3 main coronary vessels, this rules were applied: plaques in the diagonal branches were counted as belonging to the left anterior descending (LAD); plaques in the obtuse marginal an intermediate branch were counted as belonging to the LCx; plaques in the posterior descending and right postero-lateral were counted as belonging to the right coronary artery (RCA). For the last two, coronary dominance was taking into account. The ratio of "proximal-to-distal relative plaque distribution" was calculated as the proportion of plaques between these two subgroups: (1) Left main and proximal segments of the LAD, LCx and RCA; (2) Mid and distal segments of LAD and RCA, distal LCx and all evaluable coronary branches.

Definition of the coronary atherosclerotic burden indexes

The following coronary atherosclerotic burden indexes were evaluated and compared between patients with and 


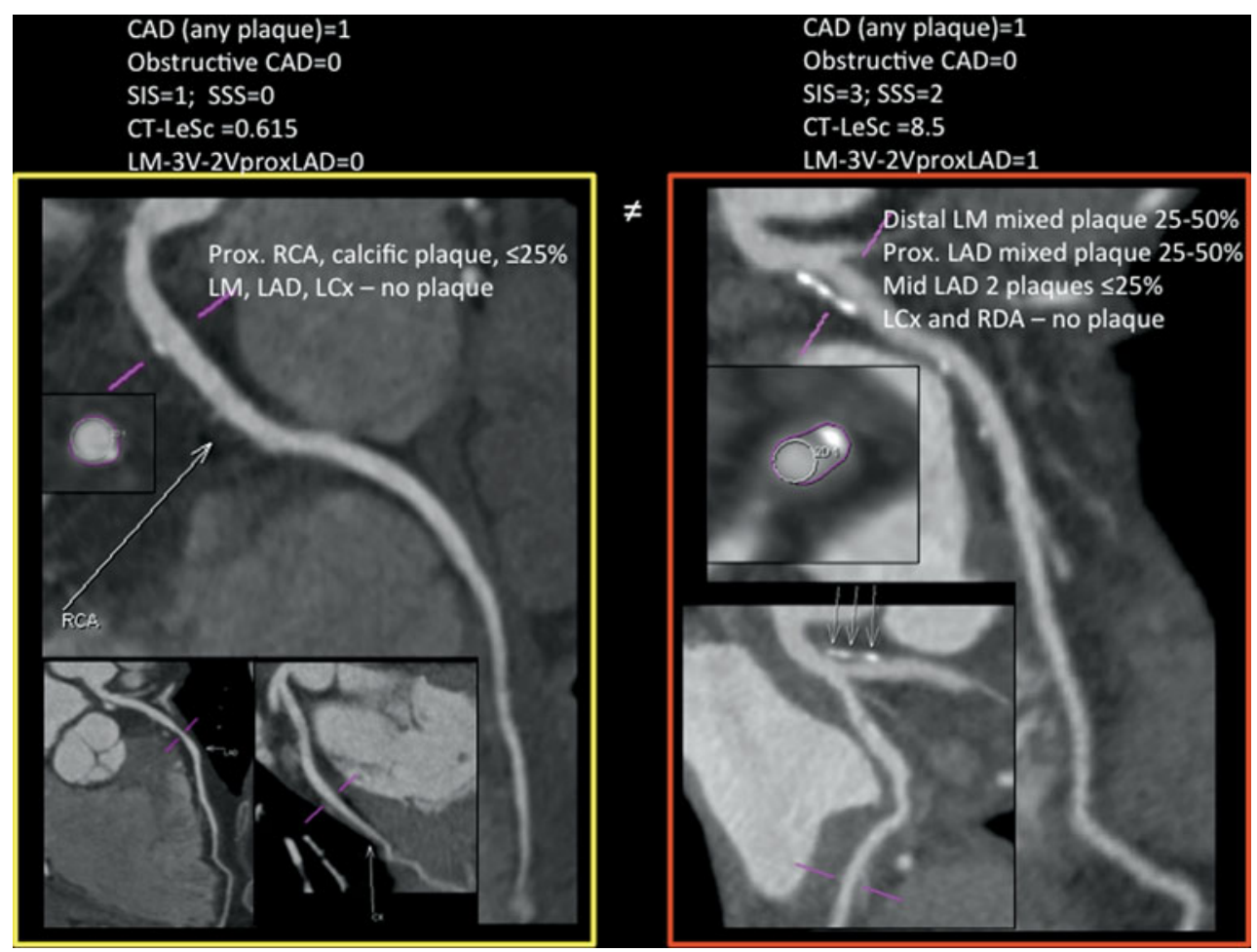

Fig. 2 Two case examples of diabetic patients with nonobstructive CAD. The different plaque burden indexes are shown. $C A D$ coronary artery disease, SIS segment involvement score, SSS segment stenosis score, $C T$-LeSc CT Leaman score, $L M$ left main, $L A D$ left anterior

without diabetes: (1) Coronary artery disease (CAD)presence of any plaque in the coronary tree; (2) "Obstructive CAD" - presence of at least one plaque with $\geq 50 \%$ stenosis; (3) "LM-3v-2v with proximal LAD"Plaque in the left main or in the 3 main epicardial vessels or in 2 main epicardial vessels including the proximal left anterior descending (LAD); (4) "SIS"-_egment involvement score, obtained as the total number of segments with plaque; (5) "SSS" —-segment stenosis score, obtained by grading the stenosis severity of each segment with plaque, as was previously described [13]. For these last two, the prognostically validated cut-offs $(>5)$ were used [13]

(6) "Calcium score (CaSc) $\geq 100 "$; (7) "CaSc $\geq 75$ th percentile" (according to published nomograms [14]; (8) CCTA-adapted Leaman score (CT-LeSc) - this score was calculated taking in account 3 weighting factors (localization, plaque composition and stenosis severity) according to previously described methodology and the same cut-off for high plaque burden $(\geq 8.3$ ) was used (provided as additional information to the reviewers, since the manuscript, where it is originally described, is under consideration elsewhere). In Fig. 2, two case examples of diabetic patients with nonobstructive CAD are shown, with the different plaque burden indexes. descending, $L C x$ left circunflex, $R C A$ right coronary artery, $L M-3 \mathrm{~V}$ 2 Vprox $L A D$ plaque in left main or 3 vessels or 2 vessels with proximal LAD

Statistical analysis

Continuous variables are presented as mean $\pm \mathrm{SD}$ or medians (interquartile range) and categorical variables as frequencies with percentages.

The non-parametric Mann-Whitney or Kruskal-Wallis tests were used to compare continuous variables. Chi square test was used to evaluate differences in frequencies. Differences were regarded significant when $p<0.05$ (twotailed).

Multivariate analyses (binary logistic regression model-enter method) were performed to identify independent predictors of CAD (any plaque and obstructive) using the demographic and clinical variables presented in Table 1, that were significant in univariate analysis $(p<0.05)$. A second multivariable analyses was performed to identify independent predictors among the clinical scores of CAD probability (Diamond-Forrester CAD consortium model and Morise score) and the $\mathrm{CV}$ risk score HeartScore. For the detailed per segment analysis, the unit of measure was each segment and there were no adjustments or corrections made for the serial correlation between segments.

SPSS version 17.0 (SPSS Inc., Chicago, IL, USA) was used for all statistical analyses. 


\section{Results}

Study population

In the final study population $(\mathrm{n}=581), 85$ patients were diabetics $(14.6 \%)$.

Regarding the demographic and clinical variables, diabetic patients were older (mean age $61.4 \pm 8.7$ vs. $56.9 \pm 11.3)$ and had a higher prevalence of obesity ( $28.6 \%$ vs. $17.4 \%$ ) and hypertension ( 84.7 vs. $58.9 \%$ ). This was predominantly a population with low to intermediate CAD probability, more so in the nondiabetic population since $61.7 \%$ had a DF-CAD consortium $<30$ and $90.7 \%$ had a Morise score $<16$. The cardiovascular risk, as estimated with the HeartScore $(\geq 5 \%)$, was significantly higher in the patients with diabetes (42.4 vs. $22.6 \%$ ) (Table 1 ).

\section{Independent predictors of CAD}

Diabetes was an independent predictor of both the presence of plaque (OR 1.81; $95 \%$ CI 1.02-3.21; $p=0.041$ ) and of obstructive CAD (OR 3.69; $95 \%$ CI 2.08-6.53; $p<0.001$ ). The other independent predictors of the presence of plaque were age $\geq 65$ years (OR 3.42; $95 \%$ CI 2.15-5.45; $p<0.001$ ), male sex (OR 2.72; $95 \%$ CI 1.85-4.01;

Table 1 Demographic and clinical characteristics of the study population

\begin{tabular}{|c|c|c|c|}
\hline & $\begin{array}{l}\text { No diabetes } \\
(\mathrm{n}=496)\end{array}$ & $\begin{array}{l}\text { Diabetes } \\
(\mathrm{n}=85)\end{array}$ & $p$ \\
\hline \multicolumn{4}{|l|}{ Demographic } \\
\hline Age & $56.9 \pm 11.3$ & $61.4 \pm 8.7$ & $<0.001$ \\
\hline Male sex & $277(55.8)$ & $47(55.3)$ & 1.000 \\
\hline \multicolumn{4}{|l|}{ Risk factors } \\
\hline Obesity (BMI $\geq 30$ ) & $85(17.4)$ & $24(28.6)$ & 0.023 \\
\hline Hypertension & $292(58.9)$ & $72(84.7)$ & $<0.001$ \\
\hline Dyslipidemia & $301(60.7)$ & $59(69.4)$ & 0.147 \\
\hline Smoking & $118(23.8)$ & $20(23.5)$ & 1.000 \\
\hline $\begin{array}{l}\text { Family history of premature } \\
\text { CAD }\end{array}$ & $168(33.9)$ & $26(30.6)$ & 0.619 \\
\hline Chest pain & $265(54.3)$ & $46(54.1)$ & 1.000 \\
\hline \multicolumn{4}{|l|}{ CAD probability } \\
\hline DF-CAD consortium $\geq 30 \%$ & $189(38.1)$ & $42(49.4)$ & 0.049 \\
\hline DF-CAD consortium $<30 \%$ & 307 (61.9) & $43(50.6)$ & \\
\hline Morise score $\geq 16$ & $46(9.3)$ & $26(30.6)$ & $<0.001$ \\
\hline Morise score 9-15 & $316(63.7)$ & $53(62.4)$ & \\
\hline Morise score $0-8$ & $134(27.0)$ & $6(7.1)$ & \\
\hline \multicolumn{4}{|l|}{$\mathrm{CV}$ risk } \\
\hline HeartScore $\geq 5 \%$ & $112(22.6)$ & $36(42.4)$ & $<0.001$ \\
\hline
\end{tabular}

Values are mean $\pm \mathrm{SD}$ or $\mathrm{n}(\%)$

$C A D$ coronary artery disease, $B M I$ body mass index, $D F-C A D$ consortium diamond-forrester $\mathrm{CAD}$ consortium model, $\mathrm{CV}$ cardiovascular $p<0.001$ ), hypertension (OR $1.82 ; 95 \%$ CI $1.23-2.67$; $p=0.002$ ), dyslipidemia (OR 1.89; $95 \%$ CI 1.29-2.77; $p=$ 0.001 ), chest pain (OR $0.62 ; 95 \%$ CI $0.42-0.91 ; p=0.014$ ) an DF-CAD consortium $\geq 30 \%$ (OR 2.62; $95 \%$ CI $1.70-4.05 ; p<0.001$ ), a Morise score $\geq 16$ (OR 2.55; $95 \%$ CI $1.57-4.14 ; p<0.001$ ), and an HeartScore $\geq 5 \%$ (OR $3.90 ; 95 \%$ CI $2.19-6.94 ; p<0.001)$. The other independent predictors of obstructive $C A D$ were age $\geq 65$ years (OR 1.98 ; $95 \%$ CI $1.16-3.37 ; p=0.012$ ), male sex (OR 2.94; $95 \%$ CI $1.68-5.15 ; p<0.001$ ), an DF-CAD consortium $\geq 30 \%$ (OR $1.88 ; 95 \%$ CI $1.04-3.42 ; p=0.038)$, a Morise score $\geq 16$ (OR 1.84; $95 \%$ CI $1.06-3.20 ; p=0.031$ ), and an HeartScore $\geq 5 \%$ (OR 2.71; $95 \%$ CI 1.50-4.88; $p=0.001)$.

Coronary artery disease prevalence, severity and coronary atherosclerotic burden indexes-per patient analysis

The prevalence of plaques in the coronary arteries was high in the overall study population, but this was significantly higher for diabetic patients, as almost 3 out of 4 diabetic patients $(74.1 \%)$ had plaques in the coronary arteries.

Table 2 Calcium score and CCTA characteristics of the study population

\begin{tabular}{llll}
\hline & $\begin{array}{l}\text { No diabetes } \\
(\mathrm{n}=496)\end{array}$ & $\begin{array}{l}\text { Diabetes } \\
(\mathrm{n}=85)\end{array}$ & $p$ \\
\hline Calcium score & $0(0-67)$ & $68(0-311)$ & $<0.001$ \\
Median & $96(19.4)$ & $40(47.1)$ & $<0.001$ \\
CaSc $\geq 100$ & $60(12.1)$ & $23(27.1)$ & 0.001 \\
CaSc $\geq 75$ th percentile & & & \\
CCTA & $217(43.8)$ & $22(25.9)$ & $<0.001$ \\
$\begin{array}{l}\text { Normal/No plaque } \\
\text { Nonobstructive CAD }\end{array}$ & $228(46.0)$ & $36(42.4)$ & \\
Obstructive CAD & $51(10.3)$ & $27(31.8)$ & \\
Coronary atherosclerotic burden indexes & & $<0.001$ \\
$\begin{array}{l}\text { Plaque in LM-3v-2v with } \\
\text { prox. LAD }\end{array}$ & $178(35.9)$ & $53(62.4)$ & \\
$\begin{array}{l}\text { Segment involvement score } \\
>5\end{array}$ & $66(13.3)$ & $31(36.5)$ & $<0.001$ \\
$\begin{array}{l}\text { Segment stenosis score }>5 \\
\text { CT-Leaman Score } \geq 8.3\end{array}$ & $25(5.0)$ & $21(24.7)$ & $<0.001$ \\
Technical data & $79(15.9)$ & $35(41.2)$ & $<0.001$ \\
$\begin{array}{l}\text { Heart rate (bpm) } \\
\text { Contrast dose (ml) }\end{array}$ & $65.3 \pm 10.6$ & $67.0 \pm 10.2$ & 0.172 \\
Radiation dose (mSv) & $99.3 \pm 14.7$ & $96.7 \pm 12.3$ & 0.119 \\
\hline
\end{tabular}

Values are mean $\pm \mathrm{SD}$, median (IQR) or $\mathrm{n}(\%)$

CaSc calcium score, CCTA coronary computed tomography angiography, $C A D$ coronary artery disease, $L M-3 v-2 v$ left main, 3 vessel, 2 vessel, $L A D$ left anterior descending, $b p m$ beats per minute, $m S v$ milisievert 
Fig. 3 Diabetes and indexes of coronary atherosclerotic burden. $C A D$ coronary artery disease, $L M$ left main, $L A D$ left anterior descending, $L C x$ left circunflex, $L M-3 V-2$ Vprox $L A D$ plaque in left main or 3 vessels or 2 vessels with proximal LAD, SIS segment involvement score, SSS segment stenosis score, $C T$ LeSc CT Leaman score

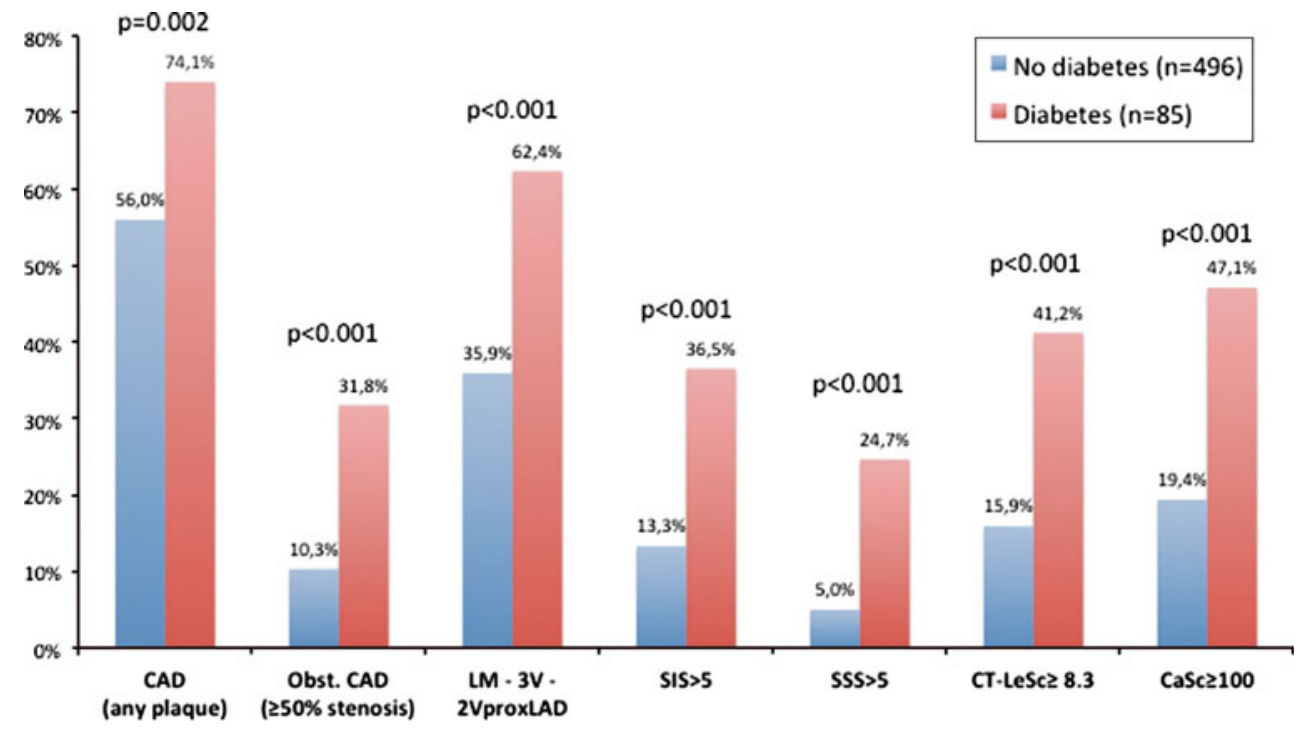

of plaque, degree of stenosis and type of plaque. Because of small size $(<2 \mathrm{~mm})$ or insufficient image quality related to artifacts or severe calcification, 866 (10.6\%) segments were excluded $(\mathrm{n}=723-10.4 \%$ in nondiabetics; $\mathrm{n}=143-12.0 \%$ in diabetics).

On a "per evaluable segment" analysis, diabetics had significantly more segments with plaque and this was observed in the left main as well as in the other 3 coronary territories and in both more proximal and more distal locations (Table 3; Fig. 5). The prevalence of obstructive plaque was also significantly higher in patients with, as compared to patients without diabetes (11.6 vs. $6.9 \%$, $p<0.001)$.

On a "per segment with plaque" analysis, nondiabetics had an almost equal distribution of plaques between more proximal (LM/proximal segments) and more mid-to-distal (Mid/distal/branches) localization (ratio of 1.04), but the opposite was seen in patients with diabetes, in whom more plaques were found in the more mid-to-distal segments, as reflected by a ratio of "proximal-to-distal relative plaque distribution" of 0.75 (Table 3).

Regarding plaque composition, diabetics had also a higher percentage of all types of plaques (both calcified and noncalcified or mixed plaques) per evaluable segment (Table 4).

\section{Discussion}

The main findings of this study are:

1. Although diabetic patients had a higher prevalence of coronary artery disease, coronary atherosclerotic plaques were commonly observed in both patients with and without diabetes. 
Table 3 Prevalence, and localization of plaques_per segment analysis

\begin{tabular}{|c|c|c|c|}
\hline & $\begin{array}{l}\text { No diabetes }(\mathrm{n}=496 \text {; } \\
6,957 \text { segments })\end{array}$ & $\begin{array}{l}\text { Diabetes }(\mathrm{n}=85 ; \\
1,187 \text { segments })\end{array}$ & $p$ \\
\hline All evaluable segments & $6,234(89.6)$ & $1,044(88.0)$ & 0.093 \\
\hline Segments with any plaque & $1,008(16.2)$ & $352(33.7)$ & $<0.001$ \\
\hline \multicolumn{4}{|l|}{ Coronary artery distribution } \\
\hline Any plaque in the LM & $87(1.4)$ & $27(2.6)$ & 0.007 \\
\hline Any plaque in the RCA & $276(4.4)$ & $110(10.5)$ & $<0.001$ \\
\hline Any plaque in the LAD & $441(7.1)$ & $137(13.1)$ & $<0.001$ \\
\hline Any plaque in the $\mathrm{LCx} / \mathrm{Ramus}$ & $204(3.3)$ & $78(7.5)$ & $<0.001$ \\
\hline \multicolumn{4}{|l|}{ Proximal versus distal distribution } \\
\hline Any plaque in LM/Proximal & $514(8.2)$ & $151(14.5)$ & $<0.001$ \\
\hline Any plaque in Mid/Distal/Branches & $494(7.9)$ & $201(19.3)$ & $<0.001$ \\
\hline "Ratio of proximal-to-distal relative plaque distribution" & $1.04(514 / 494)$ & $0.75(151 / 201)$ & 0.009 \\
\hline
\end{tabular}

"Ratio of proximal-to-distal relative plaque distribution"-proportion of plaques in the "LM/Proximal" versus "Mid/Distal/branches" $L M$ left main, $R C A$ right coronary artery, $L A D$ left anterior descending, $L C x$ left circunflex, Ramus intermediate branch, " $L M /$ Prox" left main or proximal segments of the LAD, LCx or RCA, "Mid/Distal/Branches" mid or distal segments of the LAD and RCA, distal segment of the LCx, and branches

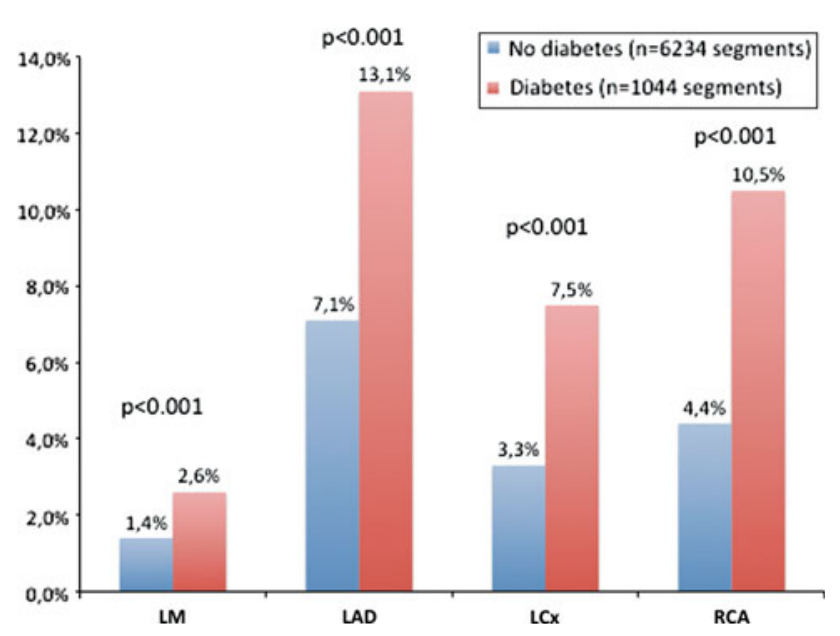

Fig. 5 Prevalence and localization of plaques (any plaque) on a per segment analysis. $L M$ left main, $L A D$ left anterior descending, $L C x$ left circunflex, $R C A$ right coronary artery
2. Several different coronary atherosclerotic burden indexes were more prevalent in diabetics, indicating more diffuse and severe $\mathrm{CAD}$, and this was especially true for males.

3. In the detailed per segment analysis, diabetics had a higher percentage of segments with plaque in every vessel and of both calcified and noncalcified or mixed types; ratio of proximal-to-distal relative plaque distribution suggested an anatomical gradient in the geographic distribution, with higher proportion of disease involvement in the $\mathrm{mid} / \mathrm{distal} / \mathrm{branches}$ segments in diabetic patients.

Diabetes as an heterogeneous group—not all diabetics have the same $\mathrm{CV}$ risk

For many years, diabetic patients have been considered as a subset at higher risk of cardiovascular events. Nevertheless,
Table 4 Type of plaques-per segment analysis

$L M$ left main, $R C A$ right coronary artery, $L A D$ left anterior descending, $L C x$ left circunflex, Ramus intermediate branch, "LM/Proximal" left main or proximal segments of the LAD, LCx or RCA, "Mid/ Distal/Branches" Mid or distal segments of the LAD and RCA, distal segment of the LCx, and branches

\begin{tabular}{llcr}
\hline & $\begin{array}{l}\text { No diabetes }(\mathrm{n}=496 ; \\
6,957 \text { segments })\end{array}$ & $\begin{array}{l}\text { Diabetes }(\mathrm{n}=85 ; \\
1,187 \text { segments })\end{array}$ & \multicolumn{1}{c}{$p$} \\
\hline $\begin{array}{l}\text { All evaluable segments } \\
\text { Segments with any plaque }\end{array}$ & $6,234(89.6)$ & $1,044(88.0)$ & 0.093 \\
Calcified plaque & $1,008(16.2)$ & $352(33.7)$ & $<0.001$ \\
All segments & & & $<0.001$ \\
LM/Proximal & $571(9.2)$ & $202(19.3)$ & $<0.001$ \\
Mid/Distal/Branches & $291(4.7)$ & $83(8.0)$ & $<0.001$ \\
Noncalcified or mixed plaques & $280(4.5)$ & $119(11.4)$ & $<0.001$ \\
All segments & $437(7.0)$ & $150(14.4)$ & $<0.001$ \\
LM/Proximal & $223(3.6)$ & $68(6.5)$ & $<0.001$ \\
Mid/Distal/Branches & $214(3.4)$ & $82(7.9)$ & \\
\hline
\end{tabular}


it has been difficult to prove a clear benefit of some primary prevention measures, like is the case of aspirin in the primary prevention of cardiovascular events. This inconsistent benefit of aspirin in the absence of clinical manifestations of cardiovascular disease, can be related to the fact that it is less effective in these patients [15] or more likely that diabetic patients are an heterogeneous group in terms of cardiovascular disease presence and extent. This way, without risk stratification, we could be overtreating some low risk diabetic patients, exposing them to the risk of side effects that could offset the reduction in expected atherothrombotic events rate.

Of note, there has been a more consistent beneficial effect of aspirin as primary preventive measure in males, for reducing the risk of myocardial infarction [3] and this is in line with our findings of higher prevalence of coronary plaques and obstructive $\mathrm{CAD}$ in this subgroup, as compared to females.

Recently, Saely et al. [16] revisited the concept of diabetes as a CAD equivalent in a study comparing the vascular event rate of patients according to the presence of diabetes and/or CAD. In this study, diabetes was not per se a CAD risk equivalent, since diabetic patients without significant $\mathrm{CAD}$ had a lower event rate than nondiabetic patients with significant $\mathrm{CAD}$.

\section{CCTA derived coronary atherosclerotic burden indexes}

Scores derived from invasive angiography have previously demonstrated to further stratify diabetic patients with more advanced CAD [17]. We hypothesized that this could also be the case for diabetics with less severe CAD, using the comprehensive information derived from CCTA on the presence, localization, degree of stenosis and plaque composition.

Several different aspects of coronary disease are reflected in these scores: prevalence and severity (any plaque and obstructive $C A D$ ), number of plaques (SIS), number and distribution (plaque in LM-3v-2v with prox. $L A D$ ), number and stenosis severity ( $S S S$ ), absolute and relative amount of calcified plaque (CaSc $\geq 100$ and $\geq 75$ th percentile) and localization, stenosis severity and type of plaque (CT-Le score). All the coronary atherosclerotic burden indexes were significantly higher in diabetics as compared to nondiabetics, reflecting the higher prevalence as well as the more severe coronary disease of this subset of patients and they can be useful as noninvasive imaging tools for risk stratification. Some of these indexes have already been prognostically validated and demonstrated a good correlation with major cardiovascular events $[13,18$, 19]. In our view, since the prevalence of plaque is very high, even in this predominantly low-to-intermediate CAD probability population, these coronary atherosclerotic burden indexes can help risk stratify patients and should ideally be included in the CCTA report. However, since they convey information on different aspects of $\mathrm{CAD}$, with some overlap in the information they provide and, in clinical practice, reporting on all of them is not suitable, ideally we should be able to decide in the future which one(s) should be routinely used, based on their prognostic performance.

Anatomical distribution and plaque composition

In this report, the higher prevalence of plaques in diabetic patients was seen in the left main as well as in the other 3 coronary territories and in both proximal and distal locations. Regarding the left main and the other proximal locations, we observed a higher percentage of plaques in diabetics as compared to nondiabetics. This is in line with previous studies linking the geographic distribution of myocardial infarction culprit lesions to more proximal locations in the coronary tree [20] and could explain the higher incidence of coronary events experienced by diabetic patients.

One interesting finding in our study is related to the relative geographic localization of plaques in diabetics as compared to patients without diabetes.

Although in prevalence of evaluable segments, diabetics had more plaques in every location (both proximal and distal) compared to nondiabetics, the relative geographic plaque distribution was different in the two subgroups of patients, since diabetics had a ratio of "proximal-to-distal relative plaque distribution" of 0.75 (vs. 1.04 for nondiabetics), suggesting a higher predisposition to disease involvement of the more distal segments. This finding, on a per segment analysis, together with the higher prevalence of a SIS $>5$ on the per patient analysis reflects the more diffuse nature of coronary atherosclerotic burden of diabetic patients.

As diabetic patients are considered to be a model of more advanced CAD, this could suggest that as the coronary atherosclerosis progresses, distal segments become more involved by disease, although serial measurements in time would be the ideal setting to evaluate this hypothesis.

The per segment analysis allowed also the evaluation of the plaque composition. Diabetic patients had a significantly higher prevalence of segments with both calcified and noncalcified or mixed plaques, in both more proximal or more distal locations. The proportion of calcified to noncalcified or mixed plaques was the same for both subgroups of patients.

\section{Limitations}

There are a number of limitations related to this report:

1. This is a single center data with medium size cohort; 
2. The population included in our study was mainly composed of patients with low to intermediate CAD probability and CV risk, as this reflects daily practice of CCTA being used as a gatekeeper to exclude obstructive CAD and are in line with the recommendations. Since coronary plaques were present in nearly $60 \%$ of the patients, this was an opportunity to evaluate the coronary atherosclerotic burden pattern of DM patients at earlier stages.

3. There were some differences in the baseline characteristics of the two subgroups of patients, that could have contributed to the higher disease extent observed in diabetic patients. Nevertheless, after adjusting for those differences, diabetes remained an independent predictor of both the presence and severity of CAD.

4. Since patients were referred for CCTA because of symptoms and/or the results of stress tests, some referral bias has to be acknowledged.

\section{Conclusions}

Diabetes was an independent predictor of CAD and was also associated with more advanced CAD, evaluated by indexes of coronary atherosclerotic burden.

The comprehensive information regarding the presence, severity and type of plaque noninvasively provided by CCTA, has made possible a detailed characterization of the coronary disease pattern of diabetic patients at an earlier stage of disease.

Diabetics had a significantly higher prevalence of plaques in every anatomical subset (type of vessel and both proximal or distal localizations) and for the different plaque composition (both calcified and noncalcified or mixed). In this report, the relative geographic distribution of the plaques within each subgroup, favored a more mid-to-distal localization in the diabetic patients.

Conflict of interest None.

\section{References}

1. Buse JB, Ginsberg HN, Bakris GL, Clark NG, Costa F, Eckel R et al (2007) Primary prevention of cardiovascular diseases in people with diabetes mellitus: a scientific statement from the American Heart Association and the American Diabetes Association. Circulation 115(1):114-126

2. Ryden L, Standl E, Bartnik M, Van den Berghe G, Betteridge J, de Boer MJ et al (2007) Guidelines on diabetes, pre-diabetes, and cardiovascular diseases: executive summary. The task force on diabetes and cardiovascular diseases of the European Society of Cardiology (ESC) and of the European Association for the Study of Diabetes (EASD). Eur Heart J 28(1):88-136
3. De Berardis G, Sacco M, Strippoli GF, Pellegrini F, Graziano G, Tognoni $G$ et al (2009) Aspirin for primary prevention of cardiovascular events in people with diabetes: meta-analysis of randomised controlled trials. BMJ 339:b4531

4. Perk J, De Backer G, Gohlke H, Graham I, Reiner Z, Verschuren $M$ et al (2012) European Guidelines on cardiovascular disease prevention in clinical practice (version 2012). The Fifth Joint Task Force of the European Society of Cardiology and Other Societies on Cardiovascular Disease Prevention in Clinical Practice (constituted by representatives of nine societies and by invited experts). Developed with the special contribution of the European Association for Cardiovascular Prevention \& Rehabilitation (EACPR). Eur Heart J 33(13):1635-1701

5. Taylor AJ, Cerqueira M, Hodgson JM, Mark D, Min J, O'Gara P et al (2010) ACCF/SCCT/ACR/AHA/ASE/ASNC/NASCI/SCAI/ SCMR 2010 appropriate use criteria for cardiac computed tomography. A report of the American College of Cardiology Foundation Appropriate Use Criteria Task Force, the Society of Cardiovascular Computed Tomography, the American College of Radiology, the American Heart Association, the American Society of Echocardiography, the American Society of Nuclear Cardiology, the North American Society for Cardiovascular Imaging, the Society for Cardiovascular Angiography and Interventions, and the Society for Cardiovascular Magnetic Resonance. J Am Coll Cardiol 56(22):1864-1894

6. (1997) Report of the Expert Committee on the Diagnosis and Classification of Diabetes Mellitus. Diabetes Care 20(7): 1183-1197

7. Expert Panel on Detection E (2001) Treatment of high blood cholesterol in A. Executive summary of the third report of the national cholesterol education program (NCEP) expert panel on detection, evaluation, and treatment of high blood cholesterol in adults (adult treatment panel III). JAMA 285(19):2486-2497

8. European Society of Hypertension-European Society of Cardiology Guidelines C (2003) European Society of HypertensionEuropean Society of Cardiology guidelines for the management of arterial hypertension. J Hypertens 21(6):1011-1053

9. Taylor AJ, Bindeman J, Feuerstein I, Cao F, Brazaitis M, O'Malley PG (2005) Coronary calcium independently predicts incident premature coronary heart disease over measured cardiovascular risk factors: mean three-year outcomes in the prospective army coronary calcium (PACC) project. J Am Coll Cardiol 46(5):807-814

10. Genders TS, Steyerberg EW, Alkadhi H, Leschka S, Desbiolles L, Nieman K et al (2011) A clinical prediction rule for the diagnosis of coronary artery disease: validation, updating, and extension. Eur Heart J 32(11):1316-1330

11. Morise AP, Haddad WJ, Beckner D (1997) Development and validation of a clinical score to estimate the probability of coronary artery disease in men and women presenting with suspected coronary disease. Am J Med 102(4):350-356

12. Raff GL, Abidov A, Achenbach S, Berman DS, Boxt LM, Budoff MJ et al (2009) SCCT guidelines for the interpretation and reporting of coronary computed tomographic angiography. J Cardiovasc Comput Tomogr 3(2):122-136

13. Min JK, Shaw LJ, Devereux RB, Okin PM, Weinsaft JW, Russo DJ et al (2007) Prognostic value of multidetector coronary computed tomographic angiography for prediction of all-cause mortality. J Am Coll Cardiol 50(12):1161-1170

14. Hoff JA, Chomka EV, Krainik AJ, Daviglus M, Rich S, Kondos GT (2001) Age and gender distributions of coronary artery calcium detected by electron beam tomography in 35,246 adults. Am J Cardiol 87(12):1335-1339

15. Evangelista V, Totani L, Rotondo S, Lorenzet R, Tognoni G, De Berardis G et al (2005) Prevention of cardiovascular disease in 
type-2 diabetes: how to improve the clinical efficacy of aspirin. Thromb Haemost 93(1):8-16

16. Saely CH, Aczel S, Koch L, Schmid F, Marte T, Huber K et al (2010) Diabetes as a coronary artery disease risk equivalent: before a change of paradigm? Eur J Cardiovasc Prev Rehabil 17(1):94-99

17. Mack MJ, Banning AP, Serruys PW, Morice MC, Taeymans Y, Van Nooten $G$ et al (2011) Bypass versus drug-eluting stents at three years in SYNTAX patients with diabetes mellitus or metabolic syndrome. Ann Thorac Surg 92(6):2140-2146

18. Andreini D, Pontone G, Mushtaq S, Bartorelli AL, Bertella E, Antonioli L et al (2012) A long-term prognostic value of coronary CT angiography in suspected coronary artery disease. JACC Cardiovasc Imaging 5(7):690-701
19. Min JK, Dunning A, Lin FY, Achenbach S, Al-Mallah M, Budoff MJ et al (2011) Age- and sex-related differences in all-cause mortality risk based on coronary computed tomography angiography findings results from the international multicenter CONFIRM (coronary CT angiography evaluation for clinical outcomes: an international multicenter registry) of 23,854 patients without known coronary artery disease. J Am Coll Cardiol 58(8):849-860

20. Wang JC, Normand SL, Mauri L, Kuntz RE (2004) Coronary artery spatial distribution of acute myocardial infarction occlusions. Circulation 110(3):278-284 